\title{
Uso de corticoides sistémicos en pacientes adultos hospitalizados por neumonía adquirida en la comunidad
}

\author{
FERNANDO TIRAPEGUI S.*, ORLANDO DÍAZ P.*y FERNANDO SALDÍAS P.*
}

\author{
Clinical efficacy of corticoids treatment in hospitalized adult patients \\ with community-acquired pneumonia
}

Community-acquired pneumonia (CAP) is a common and serious infectious disease accompanied with high morbidity and mortality. Corticosteroids (CS) therapy has been proposed for communityacquired pneumonia hospitalized adult patients. However, the effectiveness of adjunctive corticosteroids on relevant clinical outcomes of CAP remains inconsistent. Objectives: We assessed the efficacy and safety of adjunctive corticosteroids therapy in severe CAP patients. Methods: Five databases: PubMed, Scielo, Epistemonikos, Lilacs and Cochrane Library were searched for related studies published up to June, 2018. Randomized controlled trials (RCTs) of corticosteroids in hospitalized adults with severe CAP were included. Results: We assessed ten systematic reviews and fifteen primary studies enrolling severe CAP hospitalized patients. Corticosteroids therapy significantly reduced all-cause mortality (risk ratio (RR): 0.58; 95\%CI: 0.40 to 0.84), early clinical failure (RR: 0.32 ; $95 \% C I: 0.15$ to 0.7 ), risk of adult respiratory distress syndrome (ARDS) (RR: 0.23; 95\%CI: 0.07 to 0.80), need for mechanical ventilation (RR: 0.40; 95\%CI: 0.20 to 0.77) and decreased hospital length of stay (mean difference: -2.91 days; $95 \%$ CI: -4.92 to -0.89). Corticosteroids therapy increased hyperglycemia risk (RR: 1.72; 95\%CI: 1.38 to 2.14) but not gastrointestinal hemorrhage frequency (RR: 0.91; 95\%CI: 0.40 to 2.05). Conclusions: Adjuvant therapy with systemic corticosteroids decreases mortality, risk of hospital complications and shortens hospital length of stay in patients with severe CAP. These results should be confirmed by adequately powered studies in the future.

Key words: Community-acquired pneumonia, corticosteroids, efficacy, safety, prognosis, mortality.

\section{Resumen}

La neumonía adquirida en la comunidad (NAC) es una enfermedad infecciosa común y potencialmente grave que ocasiona elevada morbilidad y mortalidad. La terapia con corticosteroides (CS) sistémicos se ha propuesto para el manejo de pacientes adultos hospitalizados por neumonía adquirida en la comunidad. Objetivos: Evaluar la eficacia y seguridad del tratamiento con corticosteroides sistémicos en pacientes con NAC grave. Métodos: Se buscó la información actualizada en cinco bases de datos: PubMed, Scielo, Epistemonikos, Lilacs y Cochrane Library. Se evaluaron los ensayos clínicos controlados aleatorizados que examinaron la eficacia y seguridad de los corticosteroides en adultos hospitalizados con NAC grave. Resultados: Se incluyeron diez revisiones sistemáticas y quince estudios primarios que reclutaron pacientes hospitalizados con NAC grave. La terapia con corticosteroides redujo significativamente la mortalidad por todas las causas (cociente de riesgo [RR]: 0,58; IC95\%: 0,40 a 0,84), fracaso clínico precoz (RR: 0,32; IC95\%: 0,15 a 0,7), riesgo de síndrome de dificultad respiratoria del adulto (RR: 0,23; IC95\%: 0,07 a 0,80), necesidad de ventilación mecánica (RR: 0,40; IC95\%: 0,20 a 0,77) y se acortó la estancia hospitalaria (diferencia media: -2.91 días; IC95\%: - 4,92 a -0,89). La terapia esteroidal aumentó el riesgo de hiperglicemia (RR: 1,72; IC95\%: 1,38 a 2,14)

* Departamento de Enfermedades Respiratorias, División de Medicina, Facultad de Medicina, Pontificia Universidad Católica de Chile. Santiago, Chile. 
pero no la frecuencia de hemorragia gastrointestinal (RR: 0,91; IC95\%: 0,40 a 2,05). Conclusión: La terapia con corticosteroides sistémicos disminuye significativamente la mortalidad, riesgo de complicaciones y acorta la estancia hospitalaria en pacientes con NAC grave. Estos resultados deben ser confirmados por estudios controlados aleatorizados de mayor potencia.

Palabras clave: Neumonía adquirida en la comunidad, corticoides, eficacia, seguridad, pronóstico, mortalidad.

\section{Introducción}

La neumonía adquirida en la comunidad (NAC) es la infección respiratoria aguda que compromete el parénquima pulmonar ocasionado por microorganismos adquiridos fuera del ambiente hospitalario, correspondiendo a un proceso incubado en el medio ambiente comunitario ${ }^{1,2}$. La neumonía es un problema clínico prevalente, ocasiona morbilidad y mortalidad significativas a pesar del tratamiento antimicrobiano apropiado ${ }^{1-3}$. A pesar de los avances acontecidos en el estudio diagnóstico, tratamiento antimicrobiano y medidas de soporte, la neumonía es la principal causa de muerte de origen infeccioso en el mundo, especialmente en los niños menores de un año y adultos mayores de 65 años con comorbilidad múltiple ${ }^{1,2}$. Se estima que $20-30 \%$ de los pacientes con neumonía comunitaria son manejados en el hospital, con una letalidad que fluctúa entre $8-12 \%$ y alrededor del $10-20 \%$ son manejados en Unidades de Cuidado Intensivos con criterios de NAC severa ${ }^{1-4}$.

El tratamiento con corticosteroides (CS) sistémicos se ha recomendado en diferentes tipos de infecciones, incluyendo la meningitis bacteriana, tuberculosis, neumonía por Pneumocystis jirovecii, neumonía bacteriana y shock séptico ${ }^{5-10}$. Los mecanismos fisiopatológicos propuestos que se intentan corregir con el uso de corticosteroides difieren según el tipo de infección. En el shock séptico, una condición de estrés fisiológico extremo, se ha observado una producción inapropiada de cortisol plasmático, lo que se ha denominado insuficiencia suprarrenal asociada a la enfermedad crítica ${ }^{11}$. En la sepsis el suplemento de corticoides busca restablecer el equilibrio en el eje hipotálamo-hipófisis-suprarrenal ${ }^{6,11}$, lo cual también puede acontecer en los pacientes con neumonía comunitaria grave ${ }^{12}$. Además, los corticosteroides podrían tener un efecto beneficioso local en el sitio de la infección pulmonar. En las infecciones respiratorias, la liberación de citoquinas y otros mediadores de la inflamación de los macrófagos alveolares sería un mecanismo útil para eliminar los microorganismos patógenos. Sin embargo, la liberación excesiva de mediadores de la inflamación podría ser potencialmente dañina para el pulmón y el huésped. Los corticosteroides pueden reducir la inflamación pulmonar en casos de neumonía grave disminuyendo la disfunción pulmonar e insuficiencia respiratoria ${ }^{5,12-14}$.

En la infección respiratoria aguda, se observa un aumento significativo de las citoquinas inflamatorias circulantes, como la interleucina (IL)-6, IL-8 e IL-10, que actúan como proteínas de fase aguda, siendo su principal función regular los eventos relacionados con la respuesta inmune, ocasionando disfunción pulmonar cuando esta respuesta es exagerada ${ }^{15-17}$. Los niveles elevados de IL-6 e IL-10 circulantes se han asociado a peor pronóstico y elevada letalidad en pacientes con neumonía ${ }^{16}$, especialmente en pacientes con NAC grave podría aumentar el riesgo de sepsis, daño pulmonar y síndrome de dificultad respiratoria aguda (SDRA) ${ }^{18}$. Las citoquinas circulantes proinflamatorias pueden suprimir la respuesta del cortisol a ACTH ocasionando insuficiencia suprarrenal relativa o competir con la función del receptor de glucocorticoides intracelular determinando resistencia tisular a los corticoides ${ }^{19-21}$. De este modo, la regulación activa y efectiva del proceso inflamatorio pudiera ser vital en el manejo de los pacientes con NAC grave. Los corticosteroides, grupo de hormonas producidas en la corteza de la glándula suprarrenal, han sido implicados en una variedad de procesos fisiológicos, incluyendo la respuesta al estrés, metabolismo de los hidratos de carbono, catabolismo de las proteínas, regulación de la inflamación y la respuesta inmune, siendo reconocidos por su potente actividad antiinflamatoria ${ }^{5,6,12-14}$. Los CS inhiben la expresión de citoquinas proinflamatorias y aceleran la expresión de citoquinas antiinflamatorias ${ }^{12-14}$. La inmunomodulación y el perfil farmacodinámico antiinflamatorio han sido el fundamento fisiológico para indicar su empleo en pacientes con infección grave ${ }^{12-14}$. Además, se ha planteado que se produce insuficiencia suprarrenal aguda en el curso de algunas enfermedades críticas, siendo la terapia de reemplazo esteroidal un tratamiento recomendado en este tipo de pacientes ${ }^{11}$. Salluh y cols. midieron los niveles de cortisol basal y 
post ACTH circulantes en pacientes con NAC grave encontrando una alta prevalencia de insuficiencia adrenocortical ${ }^{22}$. Los niveles séricos de cortisol basal se correlacionaron con la gravedad de la infección pulmonar y el riesgo de muerte en el hospital. En la actualidad, se ha planteado el empleo de corticoides sistémicos en pacientes con NAC grave, no teniendo claro cuál es la dosis ni el mejor régimen de administración ${ }^{12,13,23}$. Desgraciadamente, los efectos terapéuticos de los corticoides a menudo se acompañan de efectos indeseables clínicamente significativos, los cuales dependen de la dosis, vía de administración y duración del tratamiento. En las guías de práctica clínica actuales no se recomienda el uso rutinario de corticoides sistémicos en el manejo de los pacientes con neumonía adquirida en la comunidad $^{24-26}$. El propósito de esta revisión es examinar los riesgos y beneficios asociados al tratamiento con corticoides sistémicos en pacientes hospitalizados con NAC grave.

\section{Pregunta clínica}

¿El uso de corticoides sistémicos es beneficioso o perjudicial en pacientes adultos hospitalizados por neumonía grave adquirida en la comunidad?

\section{Métodos}

Se realizó una búsqueda de los estudios primarios relevantes y revisiones sistemáticas en PubMed (US National Library of Medicine), Scielo, Epistemonikos, Lilacs, Cochrane Library y Web of Science entre enero de 1980 y junio de 2018. Las palabras clave de búsqueda y términos $\mathrm{MeSH}$ fueron community-acquired pneumoniae $A N D$ adults AND corticosteroids OR corticotherapy OR steroids OR dexamethasone OR methylprednisolone OR prednisone OR cortisone OR hydrocortisone OR prednisolone AND randomised controlled trials AND mortality.

El proceso de búsqueda y selección de artículos fue realizado por dos investigadores (FT, FS) de forma independiente y las discordancias fueron discutidas con un tercer investigador (OD), con el propósito de evitar sesgos, dar solución a las discrepancias y a la falta de información. Los artículos primarios y las revisiones sistemáticas fueron evaluados por resúmenes y título, excluyendo aquellos que no cumpliesen los criterios definidos en la pregunta clínica. El proceso de codificación y vaciamiento de información se realizó en una planilla ad hoc que contenía la información de los autores, título del estudio, fecha de publicación, diseño del estudio, fuentes de información, características de la población examinada, características de la intervención, criterios de inclusión y exclusión, estrategias de búsqueda, proceso de selección y recolección de la información, instrumentos empleados para la medición de los resultados, descripción de los resultados primarios y secundarios, síntesis de los resultados y evaluación de los riesgos de sesgo. Para ello se empleó la metodología propuesta por la organización Epistemonikos y la lista de verificación PRISMA-P recomendada para el reporte de la evidencia en una revisión sistemática ${ }^{27}$. En este proceso, se identificaron 323 publicaciones y 25 de ellas cumplieron con los criterios de la revisión.

\section{Revisión de la evidencia}

En la última década se han publicado diez revisiones sistemáticas (Tabla 1) y se identificaron quince estudios primarios que examinaron la eficacia y seguridad del uso de corticoides sistémicos en pacientes adultos hospitalizados con neumonía adquirida en la comunidad (Tabla 2).

En los últimos dos metaanálisis, Bi y cols ${ }^{36} \mathrm{y}$ Stern y $\operatorname{cols}^{3}$, evaluaron la eficacia y seguridad de los corticoides sistémicos en pacientes con NAC grave. En el primero se incluyeron ocho estudios ${ }^{40,42,43,46,47,49,50,52}$ y en el segundo trece estudios $^{39-44,46-52}$, muchos de los cuales se repitieron y cumplieron los criterios de elegibilidad definidos por los autores. Cinco estudios fueron multicéntricos ${ }^{42,48,49,51,52}$ y diez estudios fueron realizados en un solo centro ${ }^{38-41,43-47,50}$ (Tabla 2). El diagnóstico de neumonía estuvo basado en criterios clínico-radiográficos y la gravedad de la neumonía fue definida mediante los criterios de gravedad recomendados por la Sociedad Americana de Tórax, la Sociedad Británica de Tórax (CURB-65 > 2) y el índice de gravedad de la neumonía (PSI: clases IV y V) ${ }^{24-26}$. En tres estudios la intervención fue prednisona, prednisolona o hidrocortisona vía oral ${ }^{38,39,51}$ y en doce estudios fue hidrocortisona, metilprednisolona o dexametasona vía intravenosa ${ }^{40-50,52}$. Los principales resultados medidos fueron la mortalidad en el hospital y seguimiento a corto plazo, fracaso clínico, tiempo a la mejoría o curación clínica, estadía en el hospital y la UCI, admisión a UCI, uso de ventilación mecánica, las complicaciones de la neumonía y los eventos adversos asociados a la terapia esteroidal. 
Tabla 1. Revisiones sistemáticas que examinan la eficacia y seguridad del uso de corticoides sistémicos en pacientes adultos hospitalizados por neumonía adquirida en la comunidad

\begin{tabular}{|c|c|c|c|c|c|c|c|c|c|c|c|c|c|c|}
\hline \multicolumn{7}{|c|}{ Revisiones sistemáticas } & \multicolumn{8}{|c|}{ Estudios primarios } \\
\hline Salluh JI ${ }^{28}$ & 2008 & 2 & 4 & 6 & 14 & & & & & & & & & \\
\hline Siempos II $^{29}$ & 2008 & 2 & 4 & 6 & 15 & & & & & & & & & \\
\hline Nie $W^{30}$ & 2012 & 1 & 2 & 4 & 6 & 7 & 8 & 9 & 10 & 15 & & & & \\
\hline Shafiq $\mathrm{M}^{31}$ & 2013 & 1 & 2 & 4 & 6 & 7 & 8 & 9 & 10 & & & & & \\
\hline Cheng $\mathrm{M}^{32}$ & 2014 & 4 & 7 & 8 & 10 & & & & & & & & & \\
\hline Horita $\mathrm{N}^{33}$ & 2015 & 1 & 2 & 4 & 6 & 7 & 8 & 9 & 10 & 12 & 13 & & & \\
\hline Siemieniuk $\mathrm{RA}^{34}$ & 2015 & 1 & 2 & 4 & 5 & 6 & 7 & 8 & 9 & 10 & 11 & 12 & 13 & 15 \\
\hline Wan YD ${ }^{35}$ & 2016 & 2 & 4 & 6 & 7 & 8 & 9 & 11 & 12 & 13 & & & & \\
\hline $\mathrm{Bi} \mathrm{J}^{36}$ & 2016 & 2 & 4 & 5 & 7 & 8 & 10 & 11 & 13 & & & & & \\
\hline Stern $A^{37}$ & 2017 & 1 & 2 & 3 & 4 & 5 & 6 & 7 & 8 & 9 & 10 & 11 & 12 & 13 \\
\hline
\end{tabular}

Estudios primarios: 1. McHardy VU, et al. Br Med J 1972; 4: 569-73. 2. Marik P, et al. Chest 1993; 104: $389-92$.

3. Hatakeyama S, et al. Nihon Kyobu Shikkan Gakkai Zasshi 1995; 33: 51-6. 4. Confalonieri M, et al. Am J Respir Crit Care Med 2005; 171: 242-8. 5. El-Ghamrawy AH, et al. Egyptian Journal of Chest Diseases and Tuberculosis 2006; 55: 91-9. 6. Mikami K, et al. Lung 2007; 185: 249-55. 7. Snijders D, et al. Am J Respir Crit Care Med 2010; $181: 975-82$. 8. Fernández-Serrano S, et al. Crit Care 2011; 15: R96. 9. Meijvis SC, et al. Lancet 2011; 377: 2023-30. 10. Sabry NA, et al. Pharmacology \& Pharmacy 2011; 2: 73-81. 11. Nafae RM, et al. Egyptian Journal of Chest Diseases and Tuberculosis 2013; 62: 439-45. 12. Blum CA, et al. Lancet 2015; 385: 1511-8. 13. Torres A, et al. JAMA 2015; 313: 677-86. 14. García-Vidal C, et al. Eur Respir J 2007; 30: 951-6. 15. Wagner HN Jr, et al. Bull Johns Hopkins Hosp 1956; 98: $197-215$.

\section{Mortalidad por cualquier causa}

En ambas revisiones sistemáticas ${ }^{36,37}$ se evaluó el efecto de los corticoides sistémicos en la mortalidad por cualquier causa en el adulto hospitalizado por NAC grave. Se observó una reducción significativa en la mortalidad a corto plazo con el uso de CS sistémicos (cociente de riesgo (RR): 0,46; IC95\%: 0,28 a 0,77, p =0,003 y RR: 0,58 ; IC95\%: 0,40 a 0,84 respectivamente) ${ }^{36,37}$. La calidad de la evidencia fue moderada, existiendo riesgo de sesgos en la selección de las muestras. La mayoría de los ensayos clínicos emplearon dosis de CS equivalentes a $40-50 \mathrm{mg}$ de prednisona por día durante 5 a 10 días. Ninguno de los estudios proporcionó información de mortalidad en los pacientes con shock séptico o por patógenos específicos, y solo un estudio informó la mortalidad en el subgrupo con enfermedad pulmonar obstructiva crónica $(\mathrm{EPOC})^{51}$. El efecto beneficioso de los CS sistémicos fue menor en los pacientes con NAC de etiología desconocida, pero esta asociación no fue estadísticamente significativa. En un análisis de metarregresión post hoc que examinó la edad media de la población estudiada, el efecto beneficioso observado fue menor con el aumento de la edad, con un cociente de riesgo (RR) que fue aumentando en 0,05 por cada $1 \%$ de aumento en la edad media $(\mathrm{p}=0,018)$. En el estudio de Stern y $\operatorname{cols}^{37}$ se determinó el número necesario para tratar y obtener un resultado beneficioso adicional en términos de mortalidad (NNT: 18 pacientes; IC95\%: 12 a 49 pacientes).

\section{Duración de la terapia esteroidal}

En la revisión sistemática de Bi y cols ${ }^{36}$, la duración de la terapia esteroidal prolongada (mayor de 5 días) en pacientes con NAC grave demostró ser más efectivo en términos de reducción de la mortalidad (RR: 0,41; IC95\%: 0,21 a 0,82, p: $0,01)$, comparado con el curso corto de CS $(\leq 5$ días) (RR: 0,59; IC95\%: 0,24 a 1,43, p: 0,24).

\section{Falla precoz de tratamiento}

En el estudio de Stern y cols ${ }^{37}$ se logró obtener la información de 1.324 pacientes, de los cuales 419 presentaban NAC grave, logrando determinar en esta población que hubo una tasa significativamente menor de fracaso clínico temprano en los pacientes tratados con CS sistémicos en comparación con los controles (RR: 0,32; IC95\%: 0,15 a 0,7). La dosis de CS utilizada en la mayoría de los ensayos clínicos que examinaron el riesgo de fracaso clínico precoz fue equivalente a $40-50 \mathrm{mg}$ de prednisona por día durante 5 a 10 días. Solo Confalonieri y $\operatorname{cols}^{42}$ comunicaron el riesgo de fracaso clínico en el subgrupo de participantes con shock séptico. Ningún ensayo clínico examinó el riesgo de fracaso clínico según el agente causal o en pacientes con enfermedad pulmonar obstructiva crónica. 


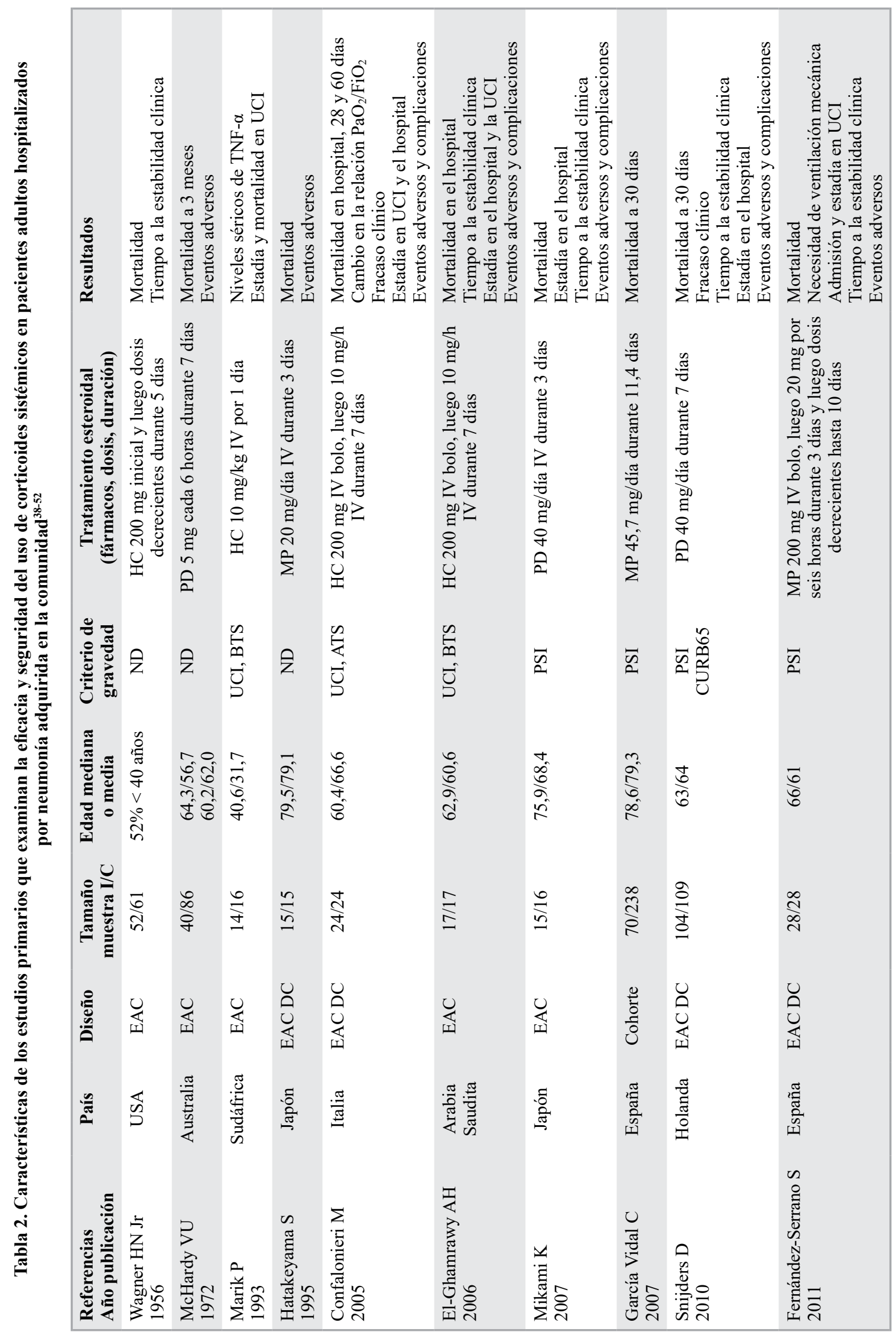




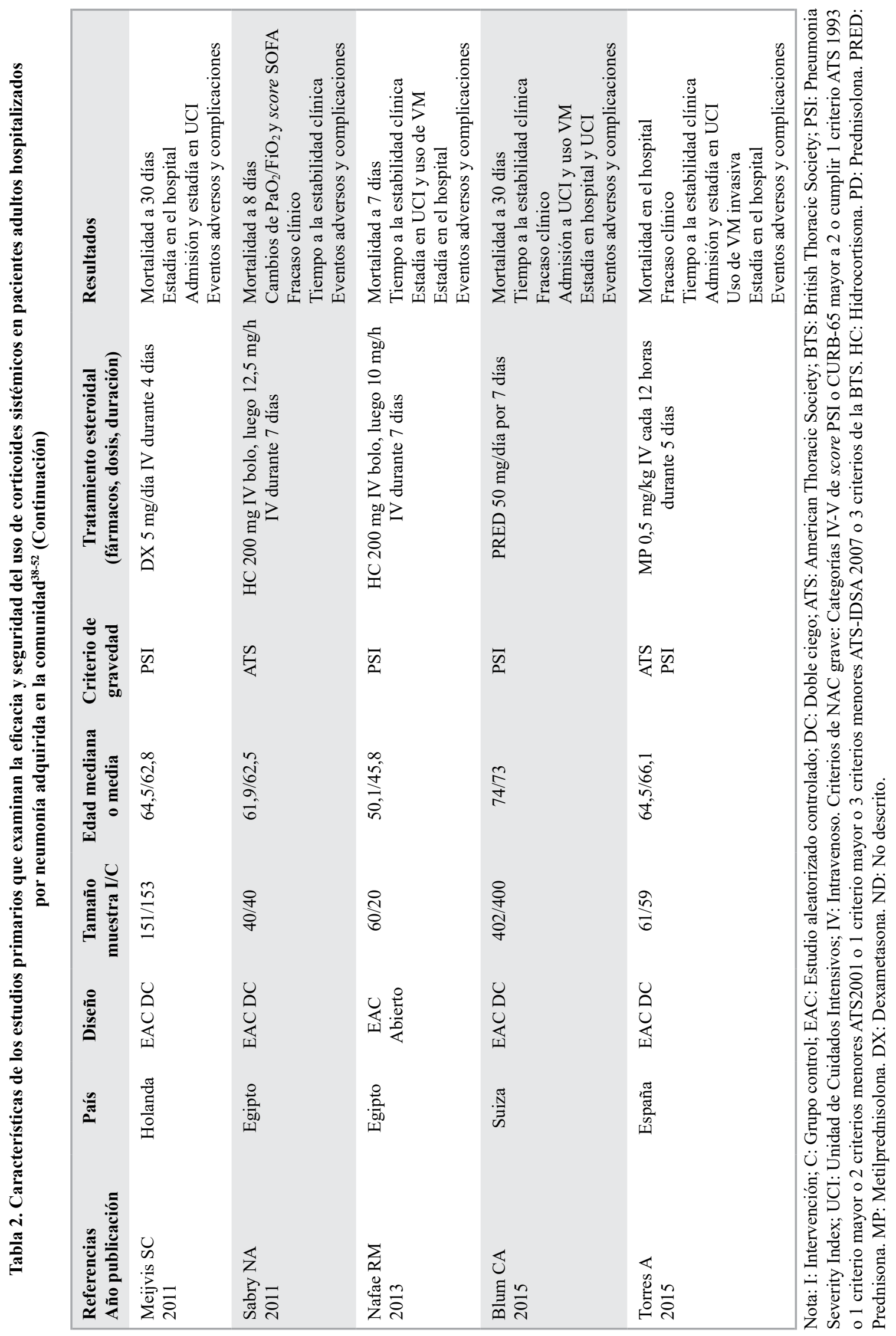


Tabla 3. Resultados de los estudios primarios que examinan la eficacia y seguridad del uso de corticoides sistémicos en pacientes adultos hospitalizados por neumonía adquirida en la comunidad ${ }^{38-52}$

Mortalidad en el hospital o seguimiento

\begin{tabular}{|c|c|c|c|c|c|}
\hline $\begin{array}{l}\text { Referencias } \\
\text { Año publicación }\end{array}$ & País & $\begin{array}{c}\text { Intervención } \\
\text { Fallecidos/Total }\end{array}$ & $\begin{array}{c}\text { Control } \\
\text { Fallecidos/Total }\end{array}$ & $\begin{array}{c}\text { Cociente de riesgo } \\
\text { (RR) }\end{array}$ & IC95\% \\
\hline $\begin{array}{l}\text { Wagner HN Jr } \\
1956\end{array}$ & USA & $1 / 52$ & $1 / 61$ & 1,17 & $0,08-18,3$ \\
\hline $\begin{array}{l}\text { McHardy VU } \\
1972\end{array}$ & Australia & $3 / 40$ & $9 / 86$ & 0,72 & $0,20-2,51$ \\
\hline $\begin{array}{l}\text { Marik P } \\
1993\end{array}$ & Sudáfrica & $1 / 14$ & $3 / 16$ & 0,38 & $0,04-3,26$ \\
\hline $\begin{array}{l}\text { Confalonieri M } \\
2005\end{array}$ & Italia & $0 / 23$ & $7 / 23$ & 0,07 & $0,001-1,10$ \\
\hline $\begin{array}{l}\text { El-Ghamrawy AH } \\
2006\end{array}$ & $\begin{array}{l}\text { Arabia } \\
\text { Saudita }\end{array}$ & $3 / 17$ & $6 / 17$ & 0,50 & $0,15-1,68$ \\
\hline $\begin{array}{l}\text { García-Vidal C } \\
2007\end{array}$ & España & & & 0,29 & $0,11-0,73$ \\
\hline $\begin{array}{l}\text { Snijders D } \\
2010\end{array}$ & Holanda & $6 / 104$ & $6 / 109$ & 1,05 & $0,35-3,15$ \\
\hline $\begin{array}{l}\text { Fernández-Serrano S } \\
2011\end{array}$ & España & $1 / 23$ & $1 / 22$ & 0,96 & $0,06-14,37$ \\
\hline $\begin{array}{l}\text { Meijvis SC } \\
2011\end{array}$ & Holanda & $9 / 151$ & $11 / 153$ & 0,83 & $0,35-1,94$ \\
\hline $\begin{array}{l}\text { Sabry NA } \\
2011\end{array}$ & Egipto & $2 / 40$ & $6 / 40$ & 0,33 & $0,07-1,55$ \\
\hline $\begin{array}{l}\text { Nafae RM } \\
2013\end{array}$ & Egipto & $4 / 60$ & $6 / 20$ & 0,22 & $0,07-0,71$ \\
\hline $\begin{array}{l}\text { Blum CA } \\
2015\end{array}$ & Suiza & $16 / 392$ & $13 / 393$ & 1,23 & $0,60-2,53$ \\
\hline $\begin{array}{l}\text { Torres A } \\
2015\end{array}$ & España & $6 / 61$ & $9 / 59$ & 0,64 & $0,24-1,70$ \\
\hline Total* & & $51 / 925$ & $77 / 938$ & 0,66 & $0,47-0,92$ \\
\hline
\end{tabular}

*Se excluyen dos estudios del análisis global de mortalidad por sesgos de selección ${ }^{38}$ y diseño del estudio (cohorte) ${ }^{45}$.

Fracaso clínico precoz

\begin{tabular}{|c|c|c|c|c|c|}
\hline $\begin{array}{l}\text { Referencias } \\
\text { Año publicación }\end{array}$ & País & $\begin{array}{c}\text { Intervención } \\
\text { Fracaso clínico/ } \\
\text { Total }\end{array}$ & $\begin{array}{c}\text { Control } \\
\text { Fracaso clínico/ } \\
\text { Total }\end{array}$ & $\begin{array}{c}\text { Cociente de riesgo } \\
\text { (RR) }\end{array}$ & IC95\% \\
\hline $\begin{array}{l}\text { Confalonieri M } \\
2005\end{array}$ & Italia & $3 / 23$ & $14 / 23$ & 0,21 & $0,07-0,65$ \\
\hline $\begin{array}{l}\text { Snijders D } \\
2010\end{array}$ & Holanda & $\begin{array}{c}13 / 48 \\
1 / 56\end{array}$ & $\begin{array}{l}12 / 45 \\
2 / 64\end{array}$ & $\begin{array}{l}1,02 \\
0,57\end{array}$ & $\begin{array}{l}0,52-1,99 \\
0,05-6,13\end{array}$ \\
\hline $\begin{array}{l}\text { Sabry NA } \\
2011\end{array}$ & Egipto & $4 / 40$ & $28 / 40$ & 0,14 & $0,06-0,37$ \\
\hline $\begin{array}{l}\text { Nafae RM } \\
2013\end{array}$ & Egipto & $4 / 60$ & $7 / 20$ & 0,19 & $0,06-0,58$ \\
\hline $\begin{array}{l}\text { Blum CA } \\
2015\end{array}$ & Suiza & $108 / 392$ & $159 / 393$ & 0,68 & $0,56-0,83$ \\
\hline $\begin{array}{l}\text { Torres A } \\
2015\end{array}$ & España & $8 / 61$ & $18 / 59$ & 0,43 & $0,20-0,91$ \\
\hline Total & & $141 / 680$ & $240 / 644$ & 0,40 & $0,23-0,70$ \\
\hline
\end{tabular}


Tabla 3. Resultados de los estudios primarios que examinan la eficacia y seguridad del uso de corticoides sistémicos en pacientes adultos hospitalizados por neumonía adquirida en la comunidad ${ }^{38-52}$ (Continuación)

Tiempo a la mejoría clínica

\begin{tabular}{|lclcccc|}
\hline $\begin{array}{l}\text { Referencias } \\
\text { Año publicación }\end{array}$ & & País & Intervención & Control & $\begin{array}{c}\text { Diferencia media } \\
\text { (días) }\end{array}$ & IC95\% \\
\hline Confalonieri M & 2005 & Italia & 15 & 19 & $-8,00$ & $-26,37$ a 10,37 \\
El-Ghamrawy AH & 2006 & $\begin{array}{l}\text { Arabia } \\
\text { Saudita }\end{array}$ & 11 & 10 & $-5,20$ & $-7,18$ a $-3,22$ \\
\hline Mikami K & 2007 & Japón & 15 & 16 & $-3,60$ & $-6,28$ a $-0,92$ \\
Snijders D & 2010 & Holanda & 104 & 109 & 0,0 & $-1,63$ a 1,63 \\
\hline Fernández-Serrano S & 2011 & España & 23 & 22 & $-2,34$ & $-4,99$ a 0,31 \\
\hline Sabry NA & 2011 & Egipto & 26 & 34 & $-2,20$ & $-2,46$ a $-1,94$ \\
Nafae RM & 2013 & Egipto & 8 & 5 & $-3,10$ & $-10,44$ a 4,24 \\
Blum CA & 2015 & Suiza & 392 & 393 & $-1,50$ & $-1,60$ a $-1,40$ \\
Torres A & 2015 & España & 61 & 59 & $-0,67$ & $-1,63$ a 0,29 \\
Total & & & 655 & 667 & $-1,83$ & $-2,45$ a $-1,21$ \\
\hline
\end{tabular}

Uso de ventilación mecánica

\begin{tabular}{|lccccc|}
\hline $\begin{array}{l}\text { Referencias } \\
\text { Año publicación }\end{array}$ & País & $\begin{array}{c}\text { Intervención } \\
\text { Uso de VM/Total }\end{array}$ & $\begin{array}{c}\text { Control } \\
\text { Uso de VM /Total }\end{array}$ & $\begin{array}{c}\text { Cociente de riesgo } \\
\text { (RR) }\end{array}$ & IC95\% \\
\hline $\begin{array}{l}\text { Fernández-Serrano S } \\
2011\end{array}$ & España & $0 / 23$ & $3 / 22$ & 0,14 & $0,01-2,51$ \\
$\begin{array}{l}\text { Nafae RM } \\
2013\end{array}$ & Egipto & $8 / 60$ & $5 / 20$ & 0,53 & $0,20-1,44$ \\
$\begin{array}{l}\text { Blum CA } \\
2015\end{array}$ & Suiza & $1 / 392$ & $6 / 393$ & 0,17 & $0,02-1,38$ \\
$\begin{array}{l}\text { Torres A } \\
2015\end{array}$ & España & $5 / 61$ & $9 / 59$ & 0,54 & $0,19-1,51$ \\
Total & & $14 / 536$ & $23 / 494$ & 0,40 & $0,20-0,77$ \\
\hline
\end{tabular}

\section{Estadía en el hospital}

\begin{tabular}{|lllcccc|}
\hline $\begin{array}{l}\text { Referencias } \\
\text { Año publicación }\end{array}$ & & País & Intervención & Control & $\begin{array}{c}\text { Diferencia media } \\
\text { (días) }\end{array}$ & IC95\% \\
\hline Confalonieri M & 2005 & Italia & 23 & 23 & $-6,67$ & $-32,75$ a 19,41 \\
El-Ghamrawy AH & 2006 & $\begin{array}{l}\text { Arabia } \\
\text { Saudita }\end{array}$ & 17 & 17 & $-6,70$ & $-10,22$ a $-3,18$ \\
Mikami K & 2007 & Japón & 15 & 16 & $-4,20$ & $-10,14$ a 1,74 \\
Snijders D & 2010 & Holanda & 104 & 109 & $-0,60$ & $-3,93$ a 2,73 \\
Fernández-Serrano S & 2011 & España & 23 & 22 & $-2,33$ & $-5,58$ a 0,92 \\
Meijvis SC & 2011 & Holanda & 151 & 153 & $-1,27$ & $-2,15$ a $-0,39$ \\
Nafae RM & 2013 & Egipto & 60 & 20 & $-7,23$ & $-8,38$ a $-6,08$ \\
Blum CA & 2015 & Suiza & 392 & 393 & $-1,40$ & $-2,09$ a $-0,71$ \\
\hline Torres A & 2015 & España & 61 & 59 & $-0,34$ & $-2,17$ a 1,49 \\
Total & & & 846 & 812 & $-2,91$ & $-4,92$ a $-0,89$ \\
\hline
\end{tabular}


Tabla 3. Resultados de los estudios primarios que examinan la eficacia y seguridad del uso de corticoides sistémicos en pacientes adultos hospitalizados por neumonía adquirida en la comunidad ${ }^{38-52}$ (Continuación)

Estadía en UCI

\begin{tabular}{|c|c|c|c|c|c|c|}
\hline $\begin{array}{l}\text { Referencias } \\
\text { Año publicación }\end{array}$ & & País & Intervención & Control & $\begin{array}{l}\text { Diferencia media } \\
\text { (días) }\end{array}$ & IC95\% \\
\hline Marik P & 1993 & Sudáfrica & 14 & 16 & $-0,30$ & $-3,81$ a 3,21 \\
\hline Confalonieri M & 2005 & Italia & 23 & 23 & $-6,33$ & $-22,70$ a 10,04 \\
\hline El-Ghamrawy AH & 2006 & $\begin{array}{l}\text { Arabia } \\
\text { Saudita }\end{array}$ & 17 & 17 & $-5,40$ & $-7,88$ a $-2,92$ \\
\hline Fernández-Serrano S & 2011 & España & 4 & 5 & $-6,75$ & $-23,27$ a 9,77 \\
\hline Meijvis SC & 2011 & Holanda & 7 & 10 & 3,47 & $-10,00$ a 16,94 \\
\hline Nafae RM & 2013 & Egipto & 60 & 20 & $-3,20$ & $-7,00$ a 0,60 \\
\hline Blum CA & 2015 & Suiza & 16 & 22 & $-2,33$ & $-6,06$ a 1,40 \\
\hline Torres A & 2015 & España & 42 & 46 & $-0,67$ & $-2,13$ a 0,79 \\
\hline Total & & & 183 & 159 & $-1,88$ & $-2,96$ a $-0,81$ \\
\hline
\end{tabular}

Riesgo de complicaciones de la neumonía

\begin{tabular}{|lllcccc|}
\hline $\begin{array}{l}\text { Referencias } \\
\text { Año publicación }\end{array}$ & & País & $\begin{array}{c}\text { Intervención } \\
\text { Complicaciones/ } \\
\text { Total }\end{array}$ & $\begin{array}{c}\text { Control } \\
\text { Complicaciones/ } \\
\text { Total }\end{array}$ & $\begin{array}{c}\text { Cociente de } \\
\text { riesgo (RR) }\end{array}$ & $\begin{array}{c}\text { IC95\% } \\
\text { Confalonieri M }\end{array}$ \\
\hline El-Ghamrawy AH & 2005 & Italia & $0 / 23$ & $1 / 23$ & 0,33 & $0,01-7,78$ \\
\hline Mikami K & 2006 & $\begin{array}{l}\text { Arabia } \\
\text { Saudita }\end{array}$ & $4 / 17$ & $10 / 17$ & 0,40 & $0,16-1,03$ \\
Snijders D & 2007 & Japón & $0 / 15$ & $0 / 16$ & ------- & -------- \\
Meijvis SC & 2010 & Holanda & $6 / 104$ & $1 / 109$ & 6,29 & $0,77-51,35$ \\
\hline Sabry NA & 2011 & Holanda & $7 / 151$ & $5 / 153$ & 1,42 & $0,46-4,37$ \\
\hline Nafae RM & 2011 & Egipto & $2 / 40$ & $6 / 40$ & 0,33 & $0,07-1,55$ \\
Blum CA & 2013 & Egipto & $4 / 60$ & $6 / 20$ & 0,22 & $0,07-0,71$ \\
\hline Total & 2015 & Suiza & $1 / 392$ & $5 / 393$ & 0,20 & $0,02-1,71$ \\
\hline
\end{tabular}

Riesgo de hiperglicemia

\begin{tabular}{|lllcccc|}
\hline $\begin{array}{l}\text { Referencias } \\
\text { Año publicación }\end{array}$ & & País & $\begin{array}{c}\text { Intervención } \\
\text { Hiperglicemia/ } \\
\text { Total }\end{array}$ & $\begin{array}{c}\text { Control } \\
\text { Hiperglicemia/ } \\
\text { Total }\end{array}$ & $\begin{array}{c}\text { Cociente de } \\
\text { riesgo (RR) }\end{array}$ & IC95\% \\
\hline Mikami K & 2007 & Japón & $0 / 15$ & $0 / 16$ & ---------- & ------- \\
\hline Snijders D & 2010 & Holanda & $5 / 104$ & $2 / 109$ & 2,62 & $0,52-13,21$ \\
\hline Fernández-Serrano S & 2011 & España & $1 / 23$ & $0 / 22$ & 2,88 & $0,12-67,03$ \\
Meijvis SC & 2011 & Holanda & $67 / 151$ & $35 / 153$ & 1,94 & $1,38-2,73$ \\
Nafae RM & 2013 & Egipto & $19 / 60$ & $8 / 20$ & 0,79 & $0,41-1,52$ \\
\hline Blum CA & 2015 & Suiza & $76 / 392$ & $43 / 393$ & 1,77 & $1,25-2,51$ \\
\hline Torres A & 2015 & España & $11 / 61$ & $7 / 59$ & 1,52 & $0,63-3,66$ \\
\hline Total & & & $179 / 806$ & $95 / 772$ & 1,72 & $1,38-2,14$ \\
\hline
\end{tabular}




\section{Tiempo a la mejoría o curación clínica}

El tiempo hasta la curación clínica fue comunicado en nueve estudios clínicos, incluyendo a 1.322 participantes. Tres ensayos clínicos ${ }^{47,51,52}$ reportaron este resultado como mediana con rango intercuartílico (IQR) y un ensayo ${ }^{42}$ informó la mediana con el rango. Para estos ensayos, los promedios y las desviaciones estándar (DE) se estimaron a partir del valor de la mediana proporcionado. El tiempo hasta la curación clínica fue significativamente más corto en el grupo tratado con CS sistémicos en comparación con el placebo (diferencia media (DM) -1,83 días; IC95\%: -2,45 a -1,21).

\section{Evolución a insuficiencia respiratoria grave (SDRA)}

Stern y cols ${ }^{37}$ evaluaron la conexión a ventilación mecánica (VM) no invasiva e invasiva, no indicada en la admisión al hospital, en cuatro estudios se evaluaron 1.030 participantes, siendo menor el riesgo de conexión a VM en el grupo tratado con CS en comparación con el grupo control (RR: 0,40; IC95\%: 0,20 a 0,77). Bi y cols ${ }^{36}$ encontraron una reducción significativa en el riesgo de SDRA en los pacientes tratado con CS (RR: 0,23; IC95\%: 0,07 a 0,80, p=0,02). Además, se observó una reducción significativa en la necesidad de ventilación mecánica en pacientes tratados con CS sistémicos (RR: 0,50; IC95\%: 0,27 a $0,92, p=0,026$ ).

\section{Evolución a shock}

En el análisis de Stern y cols ${ }^{37}$, el desarrollo de shock durante la evolución en el hospital fue examinado en cinco estudios en pacientes adultos con NAC grave y su aparición documentada fue menor en el grupo tratado con CS sistémicos en comparación con el grupo control (RR: 0,18; IC95\%: 0,09 a 0,34).

\section{Admisión a UCI}

En el estudio de Stern y $\operatorname{cols}^{37}$ se logró obtener la información de transferencia a la Unidad de Cuidados Intensivos en cuatro estudios que enrolaron a 1.164 pacientes hospitalizados por NAC. En este análisis no se encontraron diferencias significativas entre los pacientes tratados con CS sistémicos y el grupo control (RR: 0,73; IC95\%: $0,45$ a 1,18$)$.

\section{Estadía hospitalaria}

En ambas revisiones sistemáticas ${ }^{36,37}$, la estadía hospitalaria disminuyó significativamente en los pacientes con NAC grave tratados con corticoides sistémicos comparados con los grupos control (diferencia media (DM): -5,79 días; IC95\%: -8,17 a $-2,31$ y DM: $-2,91$ días; IC95\%: $-4,92$ a $-0,89$, respectivamente).

\section{Estadía en la Unidad de Cuidados Intensivos}

En la revisión sistemática de $\mathrm{Bi}$ y $\mathrm{col}^{36}$, el tratamiento con CS sistémicos no redujo significativamente la duración de la estadía en la UCI (DM: -1,84 días; IC95\%: $-4,23$ a 0,56, p: 0,13). Mientras que en el estudio de Stern y cols ${ }^{37}$, donde se evaluaron 342 pacientes con NAC grave manejados en la UCI, el tratamiento con CS sistémicos redujo significativamente la estancia en la UCI (DM: -1,88 días, IC95\%: -2,96 a -0,81).

\section{Complicaciones de la neumonía}

En la revisión sistemática de Stern y cols ${ }^{37}$, se evaluaron las complicaciones de la infección respiratoria en ocho estudios primarios que enrolaron a 1.573 pacientes, especialmente la presencia de derrame pleural, empiema y absceso pulmonar. Un ensayo clínico ${ }^{50}$ definió las complicaciones como shock séptico o síndrome de dificultad respiratoria aguda (SDRA) y dos ensayos evaluaron este resultado sin proporcionar una definición. El riesgo de complicaciones de la neumonía fue menor en los pacientes tratados con CS sistémicos en comparación con el grupo control (RR: 0,58; IC95\%: 0,40 a 0,84).

\section{Efectos adversos}

\section{Infecciones secundarias}

En la revisión sistemática de Stern y cols ${ }^{37}$ no se encontraron diferencias significativas en la tasa de infecciones secundarias entre el grupo tratado con CS sistémicos y el grupo control (RR: 1,19; IC95\%: 0,73 a 1,93 ).

\section{Hiperglicemia y hemorragia gastrointestinal}

Stern y cols ${ }^{37}$ examinaron el riesgo de eventos adversos asociados a la terapia esteroidal en siete estudios que enrolaron a pacientes con NAC grave, encontrando mayor riesgo de hiperglicemia en el grupo tratado con CS sistémicos comparado con el grupo control (RR: 1,72; IC95\%: 1,38 a 2,14), sin aumento del riesgo de hemorragia gastrointestinal (RR: 0,91; IC95\%: 0,40 a 2,05) o trastornos neuropsiquiátricos (RR: 1,95; IC95\%: 0,70 a 5,42). En el estudio de Bi y cols ${ }^{36}$, el tratamiento con CS sistémicos no aumentó el riesgo de hiperglicemia que requiriera tratamiento (RR: 1,03; IC95\%: 0,61 a 1,72, p: 0,91) ni el riesgo de hemorragia gastrointestinal (RR: 0,66; IC95\%: $0,19$ a $2,31, \mathrm{p}=0,52)$. 


\section{Conclusión}

En conclusión, la revisión de la literatura sugiere que la terapia adyuvante con corticoides sistémicos disminuye la mortalidad, riesgo de complicaciones y acorta la estadía en el hospital en pacientes con NAC grave. El tratamiento prolongado con CS sistémicos entre cinco y diez días sería beneficioso en pacientes con NAC grave. Además, el tratamiento con CS sistémicos sería bastante seguro y reduciría el riesgo de SDRA y la necesidad de ventilación mecánica. La calidad de la evidencia es moderada. Es recomendación de este grupo realizar ensayos aleatorizados, doble ciego, a gran escala controlados con placebo para evaluar la eficacia y seguridad de la terapia adyuvante de CS sistémicos en pacientes adultos con NAC grave.

\section{Referencias}

1.- REMINGTON LT, SLIGL WI. Community-acquired pneumonia. Curr Opin Pulm Med 2014; 20: 215-24.

2.- WUNDERINK RG, WATERER GW. Clinical practice. Community-acquired pneumonia. N Engl J Med 2014; 370: 543-51.

3.- ÁLVAREZ-LERMA F, TORRES A. Severe communityacquired pneumonia. Curr Opin Crit Care 2004; 10: 369-74.

4.- WOODHEAD M, WELCH CA, HARRISON DA, BELLINGAN G, AYRES JG. Community-acquired pneumonia on the intensive care unit: secondary analysis of 17,869 cases in the ICNARC Case Mix Programme Database. Crit Care 2006; 10 Suppl 2:S1.

5.- MONTÓN C, EWIG S, TORRES A, EL-EBIARY M, FILELLA X, RAÑó A, et al. Role of glucocorticoids on inflammatory response in nonimmunosuppressed patients with pneumonia: a pilot study. Eur Respir J 1999; 14: 218-20.

6.- ANNANE D, BELLISSANT E, BOLLAERT PE, BRIEGEL J, CONFALONIERI M, DE GAUDIO R, et al. Corticosteroids in the treatment of severe sepsis and septic shock in adults: a systematic review. JAMA 2009; 301: 2362-75.

7.- CRITCHLEY JA, YOUNG F, ORTON L, GARNER P. Corticosteroids for prevention of mortality in people with tuberculosis: a systematic review and meta-analysis. Lancet Infect Dis 2013; 13: 223-37.

8.- ANNANE D, BELLISSANT E, BOLLAERT PE, BRIEGEL J, KEH D, KUPFER Y. Corticosteroids for treating sepsis. Cochrane Database Syst Rev 2015; (12): CD002243.

9.- EWALD H, RAATZ H, BOSCACCI R, FURRER H, BUCHER HC, BRIEL M. Adjunctive corticosteroids for Pneumocystis jiroveci pneumonia in patients with
HIV-infection. Cochrane Database Syst Rev 2015; (4): CD006150.

10.- BROUWER MC, MCINTYRE P, PRASAD K, VAN DE BEEK D. Corticosteroids for acute bacterial meningitis. Cochrane Database Syst Rev 2015; (9): CD004405.

11.- MARIK PE, PASTORES SM, ANNANE D, MEDURI GU, SPRUNG CL, ARLT W, et al. Recommendations for the diagnosis and management of corticosteroid insufficiency in critically ill adult patients: consensus statements from an international task force by the American College of Critical Care Medicine. Crit Care Med 2008; 36: 1937-49.

12.- SIBILA O, AGUSTÍ C, TORRES A. Corticosteroids in severe pneumonia. Eur Respir J 2008; 32: 259-64.

13.- ARIANI F, LIU K, JING Z, QU J. Glucocorticosteroid in treatment of severe pneumonia. Mediators Inflamm 2013; 2013: 865635.

14.- RHEN T, CIDLOWSKI JA. Antiinflammatory action of glucocorticoids - new mechanisms for old drugs. N Engl J Med 2005; 353: 1711-23.

15.- KELLY E, MACREDMOND RE, CULLEN G, GREENE CM, MCELVANEY NG, O'NEILL SJ. Communityacquired pneumonia in older patients: does age influence systemic cytokine levels in community-acquired pneumonia? Respirology 2009; 14: 210-6.

16.- MARTÍNEZ R, MENÉNDEZ R, REYES S, POLVERINO E, CILLÓNIZ C, MARTÍNEZ A, et al. Factors associated with inflammatory cytokine patterns in community-acquired pneumonia. Eur Respir J 2011; 37 : 393-9.

17.- ENDEMAN H, MEIJVIS SC, RIJKERS GT, VAN VELZEN-BLAD H, VAN MOORSEL CH, GRUTTERS JC, et al. Systemic cytokine response in patients with community-acquired pneumonia. Eur Respir J 2011; 37: 1431-8.

18.- ALIBERTI S, BRAMBILLA AM, CHALMERS JD, CILLONIZ C, RAMÍREZ J, BIGNAMINI A, et al. Phenotyping community-acquired pneumonia according to the presence of acute respiratory failure and severe sepsis. Respir Res 2014; 15: 27.

19.- JÄÄTTELÄ M, ILVESMAKI V, VOUTILAINEN R, STENMAN UH, SAKSELA E. Tumor necrosis factor as a potent inhibitor of adrenocorticotropin-induced cortisol production and steroidogenic P450 enzyme gene expression in cultured human fetal adrenal cells. Endocrinology 1991; 128: 623-9.

20.- ANNANE D, SÉBILLE V, TROCHÉ G, RAPHAEL JC, GAJDOS P, BELLISSANT E. A 3-level prognostic classification in septic shock based on cortisol levels and cortisol response to corticotropin. JAMA 2000; 283 : 1038-45.

21.- MOLIJN GJ, KOPER JW, VAN UFFELEN CJ, DE JONG FH, BRINKMANN AO, BRUINING HA, et al. Temperature-induced down-regulation of the glucocorticoid receptor in peripheral blood mononuclear leucocyte 
in patients with sepsis or septic shock. Clin Endocrinol (Oxf) 1995;43:197-203.

22.- SALLUH JIF, BOZZA FA, SOARES M, VERDEAL JCR, CASTRO-FARIA-NETO HC, LAPA E, et al. Adrenal response in severe community-acquired pneumonia: impact on outcomes and disease severity. Chest 2008; 134: 947-54.

23.- TAGAMI T, MATSUI H, HORIGUCHI H, FUSHIMI K, YASUNAGA H. Low-dose corticosteroid use and mortality in severe community-acquired pneumonia patients. Eur Respir J 2015;45: 463-72.

24.- MANDELL LA, WUNDERINK RG, ANZUETO A, BARTLETT JG, CAMPBELL GD, DEAN NC, et al. Infectious Diseases Society of America/American Thoracic Society Consensus Guidelines on the management of community-acquired pneumonia in adults. Clin Infect Dis 2007; 44 (Suppl 2): S27-S72.

25.- LIM WS, BAUDOUIN SV, GEORGE RC, HILL AT, JAMIESON C, LE JEUNE I, et al; Pneumonia Guidelines Committee of the BTS Standards of Care Committee. The British Thoracic Society Guidelines for the management of community-acquired pneumonia in adults: update 2009. Thorax 2009; 64 (Suppl 3): 1-55.

26.- BANTAR C, CURCIO D, JASOVICH A, BAGNULO H, ARANGO A, BAVESTRELLO L, et al. Neumonía aguda adquirida en la comunidad en adultos: Actualización de los lineamientos para el tratamiento antimicrobiano inicial basado en la evidencia local del Grupo de Trabajo de Sudamérica (ConsenSur II). Rev Chil Infect 2010; 27 (Supl 1): 9-38.

27.- SHAMSEER L, MOHER D, CLARKE M, GHERSI D, LIBERATI A, PETTICREW M, et al; PRISMA-P Group. Preferred reporting items for systematic review and meta-analysis protocols (PRISMA-P) 2015: elaboration and explanation. BMJ 2015; 350: g7647.

28.- SALLUH JI, PÓVOA P, SOARES M, CASTROFARIA-NETO HC, BOZZA FA, BOZZA PT. The role of corticosteroids in severe community-acquired pneumonia: a systematic review. Crit Care 2008; 12: R76.

29.- SIEMPOS II, VARDAKAS KZ, KOPTERIDES P, FALAGAS ME. Adjunctive therapies for communityacquired pneumonia: a systematic review. J Antimicrob Chemother 2008; 62: 661-8.

30.- NIE W, ZHANG Y, CHENG J, XIU Q. Corticosteroids in the treatment of community- acquired pneumonia in adults: a meta-analysis. PLoS One 2012; 7: e47926.

31.- SHAFIQ M, MANSOOR MS, KHAN AA, SOHAIL MR, MURAd MH. Adjuvant steroid therapy in community-acquired pneumonia: A systematic review and meta-analysis. J Hosp Med 2013; 8: 68-75.

32.- CHENG M, PAN ZY, YANG J, GAO YD. Corticosteroid therapy for severe community-acquired pneumonia: a meta-analysis. Respir Care 2014; 59: 557-63.

33.- HORITA N, OTSUKA T, HARANAGA S, NAMKOONG H, MIKI M, MIYASHITA N, et al. Adjunctive systemic corticosteroids for hospitalized community-acquired pneumonia: Systematic review and meta-analysis 2015 update. Sci Rep 2015;5:14061.

34.- SIEMIENIUK RA, MEADE MO, ALONSO-COELLO P, BRIEL M, EVANIEW N, PRASAD $M$, et al. Corticosteroid therapy for patients hospitalized with community-acquired pneumonia. A systematic review and meta-analysis. Ann Intern Med 2015; 163: 519-28.

35.- WAN YD, SUN TW, LIU ZQ, ZHANG SG, WANG LX, KAN QC. Efficacy and safety of corticosteroids for community-acquired pneumonia. A systematic review and meta-analysis. Chest 2016; 149: 209-19.

36.- BI J, YANG J, WANG Y, YAO C, MEI J, LIU Y, et al. Efficacy and safety of adjunctive corticosteroids therapy for severe community-acquired pneumonia in adults: An updated systematic review and meta-analysis. PLoS One 2016; 11: e0165942.

37.- STERN A, SKALSKY K, AVNI T, CARRARA E, LEIBOVICI L, PAUL M. Corticosteroids for pneumonia. Cochrane Database Syst Rev 2017; 12: CD007720.

38.- WAGNER HN JR, BENNETT IL JR, LASAGNA L, CLUFF LE, ROSENTHAL MB, MIRICK GS. The effect of hydrocortisone upon the course of pneumococcal pneumonia treated with penicillin. Bull Johns Hopkins Hosp 1956; 98: 197-215.

39.- MCHARDY VU, SCHONELL ME. Ampicillin dosage and use of prednisolone in treatment of pneumonia: cooperative controlled trial. Br Med J 1972; 4: 569-73.

40.- MARIK P, KRAUS P, SRIBANTE J, HAVLIK I, LIPMAN J, JOHNSON DW. Hydrocortisone and tumor necrosis factor in severe community-acquired pneumonia. A randomized controlled study. Chest 1993; 104: 389-92.

41.- HATAKEYAMA S, TACHIBANA A, SUZUKI K, OKANO H. Treatment of aspiration pneumonia with low-dose methylprednisolone and antibiotics. Nihon Kyobu Shikkan Gakkai Zasshi 1995; 33: 51-6.

42.- CONFALONIERI M, URBINO R, POTENA A, PIATTELLA M, PARIGI P, PUCCIO G, et al. Hydrocortisone infusion for severe community-acquired pneumonia: a preliminary randomized study. Am J Respir Crit Care Med 2005; 171: 242-8.

43.- EL-GHAMRAWY AH, SHOKIER MH, ESMAT AA. Effects of low-dose hydrocortisone in ICU patients with severe community-acquired pneumonia. Egyptian Journal of Chest Diseases and Tuberculosis 2006; 55 91-9.

44.- MIKAMI K, SUZUKI M, KITAGAWA H, KAWAKAMI M, HIROTA N, YAMAGUCHI H, et al. Efficacy of corticosteroids in the treatment of community-acquired pneumonia requiring hospitalization. Lung 2007; 185: 249-55.

45.- GARCÍA-VIDAL C, CALBO E, PASCUAL V, FERRER C, QUINTANA S, GARAU J. Effects of systemic steroids in patients with severe community-acquired pneumonia. Eur Respir J 2007; 30: 951-6.

46.- SNIJDERS D, DANIELS JM, DE GRAAFF CS, VAN 
DER WERF TS, BOERSMA WG. Efficacy of corticosteroids in community-acquired pneumonia: a randomized double-blinded clinical trial. Am J Respir Crit Care Med 2010; 181: 975-82.

47.- FERNÁNDEZ-SERRANO S, DORCA J, GARCÍAVIDAL C, FERNÁNDEZ-SABÉ N, CARRATALÀ J, FERNÁNDEZ-AGÜERA A, et al. Effect of corticosteroids on the clinical course of community-acquired pneumonia: a randomized controlled trial. Crit Care 2011; 15: R96.

48.- MEIJVIS SC, HARDEMAN H, REMMELTS HH, HEIJLIGENBERG R, RIJKERS GT, VAN VELZENBLAD H, et al. Dexamethasone and length of hospital stay in patients with community-acquired pneumonia: a randomised, double-blind, placebo-controlled trial. Lancet 2011; 377: 2023-30.

49.- SABRY NA, OMAR EE. Corticosteroids and ICU course of community acquired pneumonia in Egyptian settings. Pharmacology \& Pharmacy 2011; 2: 73-81.

50.- NAFAE RM, RAGAB MI, AMANY FM, RASHED SB. Adjuvant role of corticosteroids in the treatment of community-acquired pneumonia. Egyptian Journal of Chest Diseases and Tuberculosis 2013; 62: 439-45.

51.- BLUM CA, NIGRO N, BRIEL M, SCHUETZ P, ULLMER E, SUTER-WIDMER I, et al. Adjunct prednisone therapy for patients with community-acquired pneumonia: a multicentre, double-blind, randomised, placebocontrolled trial. Lancet 2015; 385: 1511-8.

52.- TORRES A, SIBILA O, FERRER M, POLVERINO E, MENÉNDEZ R, MENSA J, et al. Effect of corticosteroids on treatment failure among hospitalized patients with severe community-acquired pneumonia and high inflammatory response: a randomized clinical trial. JAMA 2015; 313: 677-86.
Correspondencia a:

Dr. Fernando Saldías Peñafiel

Departamento de Enfermedades Respiratorias, División de Medicina,

Facultad de Medicina, Pontificia Universidad Católica de Chile.

Diagonal Paraguay 362, Sexto Piso, Santiago, Chile.

Email: fsaldias@med.puc.cl 